\title{
A perioperative infusion of sodium bicarbonate does not improve renal function in cardiac surgery patients: a prospective observational cohort study
}

Matthias Heringlake ${ }^{1 *}$, Hermann Heinze ${ }^{1}$, Maria Schubert ${ }^{1}, Y_{\text {vonne Nowak}}{ }^{1}$, Janina Guder ${ }^{1}$, Maria Kleinebrahm', Hauke Paarmann ${ }^{1}$, Thorsten Hanke ${ }^{2}$ and Julika Schön ${ }^{1}$

\begin{abstract}
Introduction: Cardiac-surgery-associated-acute-kidney-injury (CSA-AKI) is associated with increased morbidity and mortality. Recent data from patients undergoing on-pump coronary artery bypass grafting suggest that a perioperative infusion of sodium-bicarbonate may decrease the incidence of CSA-AKI. The present study aims to analyze the renoprotective effects of a $24 \mathrm{~h}$ infusion of sodium-bicarbonate in a large, heterogeneous group of cardiac surgical patients

Methods: Starting in 4/2009, all patients undergoing cardiac surgery at our institution were enrolled in a prospective trial analyzing the relationship between preoperative cerebral oxygen saturation and postoperative organ dysfunction. We used this prospectively sampled data set to perform a cohort analysis of the renoprotective efficiency of a $24 \mathrm{~h}$ continuous perioperative infusion of sodium-bicarbonate on the incidence of CSA-AKI that was routinely introduced in 7/2009. After exclusion of patients with endstage chronic kidney disease, off-pump procedures, and emergency cases, perioperative changes in renal function were assessed in 280 patients treated with a perioperative infusion of $4 \mathrm{mmol}$ sodium-bicarbonate / $\mathrm{kg}$ body weight in comparison with a control cohort of 304 patients enrolled from April to June in this prospective cohort study.

Postoperative changes in urine flow, plasma creatinine, estimated creatinine clearance, and the need for renal replacement therapy were determined according to AKI injury network criteria. Concomitantly, hemodynamics, treatments, complications, and clinical outcomes were recorded. Univariate statistical analyses were performed para- and nonparametrically, as appropriate.

Results: With the exception of a lower prevalence of a history of myocardial infarction and a lower preoperative use of intravenous heparin in the bicarbonate-group, no significant between group differences in patient demographics, surgical risk, type, and duration of surgery were observed. Patients in the bicarbonate group had a lower mean arterial blood pressure after induction of anesthesia, needed more fluids, more vasopressors, and a longer treatment time in the high dependency unit. Despite a higher postoperative diuresis, no differences in the incidence of AKI grade 1 to 3 and the need for renal replacement were observed.
\end{abstract}

Conclusions: Routine perioperative administration of sodium bicarbonate failed to improve postoperative renal function in a large population of cardiac surgical patients.

\footnotetext{
* Correspondence: Heringlake@t-online.de

'Department of Anesthesiology, University of Lübeck, Ratzeburger Allee 160,

Lübeck, D-23538, Germany

Full list of author information is available at the end of the article
} 


\section{Introduction}

Acute kidney injury (AKI) is a frequent complication in patients undergoing cardiac surgery [1,2]. With respect to the multifactorial nature of this complication and the consequences for short- and long-term prognosis [3] there is an increasing clinical and scientific interest in this topic that has now been specifically referred to as cardiac surgery-associated kidney injury (CSA-AKI) [4].

Recent work suggests that the renal excretion of hemoand myoglobin, as a consequence of the destruction of blood cells during cardiopulmonary bypass and tissue injury during prolonged periods of hypoperfusion, and the subsequent development of a pigment nephropathy, may play a pathophysiologically important role in CSAAKI [5]. In support of this concept and with respect to the fact that urine alkalization with sodium bicarbonate (BIC) has traditionally been used to ameliorate the consequences of hemo- and myoglobinuria [6], Haase and coworkers have recently shown that perioperative $24-\mathrm{h}$ treatment with BIC reduced the incidence of AKI in a double-blind pilot trial including 100 patients undergoing on-pump cardiac surgery [7]; findings that are currently being prospectively validated in 500 patients (the BICNC study; Clinical trials identifier NCT00672334)

With respect to the enormous impact of postoperative renal dysfunction on outcome in cardiac surgical patients, the sound pathophysiological basis, and positive historical experiences with urine alkalization in our center we decided not to await the results of the ongoing multicentre trial and implemented the concept of perioperative BIC infusion in July 2009 at our institution. With respect to the fact that since April 2009 almost all patients undergoing cardiac surgery at the University of Lübeck were enrolled in a prospective observational trial analyzing the association between preoperative cerebral oxygen saturation and postoperative organ dysfunction, we chose to use this database also to analyze the effects of routine BIC application on the perioperative changes in renal function and the incidence of AKI in this prospective, observational cohort study.

\section{Materials and methods}

Following approval by the local ethical committee (Ethikkommission der Universität zu Lübeck, Lübeck, Germany), all patients scheduled for cardiac surgery at the University of Lübeck from 1 April 2009 to 31 December 2009 were screened for participation in a prospective, observational trial on the relationship between preoperative cerebral oxygenation and postoperative organ dysfunction. The only exclusion criterion was age less below 18 years. Written informed consent was obtained from all elective and urgent patients as well as emergency patients who were able to communicate. In the case of sedated and/or intubated patients scheduled for emergency surgery, consent was obtained from the next of kin and reconfirmed after recovery.

From July 2009, all patients were treated with a perioperative infusion of $\mathrm{BIC}$, and the treatment was maintained until the first results of the present study were available in December 2010. Following approval by the local ethical committee of amendment 5 to the primary request (reference number, 07-146), we specifically analyzed the perioperative changes in renal function and general patient outcomes according to the use or non-use of BIC. After exclusion of patients with end-stage chronic kidney disease, off-pump procedures, and emergency cases, perioperative changes in renal function were assessed in 280 patients treated with $\mathrm{BIC}$ in comparison with a control cohort $(\mathrm{CON})$ of 304 patients enrolled from April to June. In addition to the standard treatment, plasma samples for determination of N-terminal pro B-type natriuretic peptide (NTproBNP) and high-sensitivity troponin-T (hsTNT) were drawn immediately before surgery.

The perioperative infusion of BIC was adapted from Haase and coworkers [(7)]. A bolus of $0.5 \mathrm{mmol} / \mathrm{Kg}$ body weight $(\mathrm{BW})$ in a balanced electrolyte infusion $(500 \mathrm{ml})$ was infused within 30 minutes immediately after induction of anesthesia. Thereafter, a maintenance infusion of $0.15 \mathrm{mmol} / \mathrm{Kg} \mathrm{BW} / \mathrm{h}$ in $500 \mathrm{ml}$ dextrose in water (5\%) was applied within $24 \mathrm{~h}$, giving a total dose of $4 \mathrm{mmol} \mathrm{BIC/Kg} \mathrm{BW}$ during $24 \mathrm{~h}$. Anesthesiological, surgical, and intensive care treatment followed the standardized algorithms established at the Department of Anesthesiology and the Department of Thoracic Vascular and Cardiac Surgery of the University of Lübeck.

\section{Anesthesiological and intensive care treatments}

Following oral premedication with 3.75 to $7.5 \mathrm{mg}$ midazolam, and transfer to the operating room, sensors for the determination of cerebral oxygen saturation $(\mathrm{ScO} 2)$ with an INVOS ${ }^{\circledR} 5100$ monitor (Somanetics, Troy, MI, USA) were applied bi-hemisperically before induction of anesthesia. Baseline $\mathrm{ScO} 2$ was determined in the resting state when breathing room air. General anesthesia was induced with sufentanil $0.5 \mu \mathrm{g} / \mathrm{Kg} \mathrm{BW}$, etomidate 0.2 to $0.4 \mathrm{mg} / \mathrm{Kg} \mathrm{BW}$ and maintained at 0.8 to 1.0 minimal alveolar concentration of sevoflurane and remifentanyl at 0.2 to $0.4 \mu \mathrm{g} / \mathrm{Kg} \mathrm{BW} / \mathrm{min}$, with the goal of early postoperative extubation. Muscle relaxation was achieved with rocuronium bromide $0.6 \mathrm{mg} / \mathrm{Kg} \mathrm{BW}$. During cardiopulmonary bypass (CPB), propofol at a dose of 4 to $5 \mathrm{mg} / \mathrm{Kg}$ $\mathrm{BW} / \mathrm{h}$ was applied, since at that time volatile anesthetics could not be given via the CPB circuit. After surgery, all patients were transferred to the ICU, and after normothermia had been achieved, were weaned from the respirator as soon as possible. 
Hemodynamic therapy aimed at a mean arterial blood pressure (MAP) between 60 and $90 \mathrm{mmHg}$, a heart rate (HR) between 60 and $90 \mathrm{bpm}$, a central venous pressure (CVP) between 10 and $15 \mathrm{mmHg}$, and central venous oxygen saturation $(\mathrm{ScvO} 2)$ greater than $70 \%$. In patients monitored with a pulmonary artery catheter, hemodynamic therapy was titrated to achieve a cardiac index $>2.2 \mathrm{l} /$ $\mathrm{min} / \mathrm{m} 2$ and mixed venous oxygen saturation (SvO2) greater than 65\% [8]. Pulmonary arterial pressure (PAP) was used to guide hemodynamic therapy for right heart and pulmonary vascular function.

Fluid therapy was performed by balanced cristalloid (Sterofundin ISO 1/1; BBraun; Melsungen, Germany) and colloidal (Volulyte ${ }^{\circledR}$, Fresenius, Germany; Gelafun$\operatorname{din}^{\circledR}$, BBraun, Melsungen, Germany) fluids, as suggested by a recent guideline [8].

\section{Surgical and cardiopulmonary bypass treatments}

All surgeries were performed with $\mathrm{CPB}$ in moderate or deep hypothermia (according to the preferences of the surgeon and the scheduled type of surgery). Cardioplegic arrest was achieved by blood cardioplegia and repeated every 20 minutes. In coronary-artery bypass grafting (CABG), a single crossclamp was generally used. Non-pulsatile perfusion was performed during CPB. Pump flow, oxygen flow, and MAP were adjusted to maintain $\mathrm{ScO} 2$ levels higher than $50 \%$ absolute.

\section{Clinical treatments and outcomes}

Patients were discharged from the ICU to the intermediate care unit (IMC) if they were hemodynamically stable with only moderate inotropic and/or vasopressor support and were breathing spontaneously without the need for noninvasive ventilation. They were discharged to the surgical ward if they had been completely weaned from vasoactive or inotropic drugs, showed no signs of a relevant organ dysfunction (that is, pneumonia, AKI, severe neurological dysfunction, etcetera), and were free of delirium.

A perioperative myocardial infarction was defined as a relevant increase in myocardial necrosis markers (creatinine-kinase and creatinine-kinase MB fraction) in combination with the development of typical electrocardiographic findings or the detection of a new wall motion abnormality by echocardiography.

Indication and performance of renal replacement therapy Indications for renal replacement therapy were as follows: potassium equal to or higher than $6 \mathrm{mmol} / \mathrm{L}$ despite forced diuresis or prolonged treatment with glucoseinsulin solution; fluid overload with imminent or present extrarenal organ dysfunction not responsive to moderate doses of diuretics (that is, more than $80 \mathrm{mg} / \mathrm{d}$ torasemide), severe low cardiac output state with persistent oliguria despite, or inadequate reaction to a bolus of a loop diuretic (furosemide $40 \mathrm{mg}$, torasemide $20 \mathrm{mg}$ ).

Renal replacement therapy was performed as venovenous hemodiafiltration in postdilution mode with a blood flow of 150 to $200 \mathrm{ml} / \mathrm{min}$, an ultrafiltration dose of $20 \mathrm{ml} / \mathrm{Kg} \mathrm{BW} / \mathrm{h}$, and a dialysis dose of $15 \mathrm{ml} / \mathrm{Kg} / \mathrm{h}$.

\section{Statistical analysis}

Data entry and analysis were performed with MedCalc 12.1.4. for Windows. Following Kolmogorov-Smirnov testing for normality of distribution, data were analyzed by parametric and non-parametric tests, as appropriate. Accordingly, if not stated otherwise, data are presented as mean and 95\% confidence interval for the mean, or median and $95 \%$ confidence interval for the median. A $P$-value < 0.05 was considered to indicate statistical significance.

The severity of postoperative kidney dysfunction was quantified according to the criteria of AKI as suggested by the Acute Kidney Injury Network [9]. Additionally, and with respect to the ongoing prospective trial on the effects of BIC on renal function in cardiac surgery patients (BIC-NC study; Clinical trials identifier NCT00672334), we determined the number of patients showing a postoperative increase in plasma creatinine between 25 and $50 \%$.

\section{Results}

\section{Comparability of the study cohorts}

With the exception of a lower prevalence of a history of myocardial infarction (18.6\% vs. $26.3 \%)$ and a lower preoperative use of intravenous heparin in the BIC group, the study cohorts were highly comparable regarding baseline demographics, surgical procedures, and surgical core data (Table 1, 2, and 3). No significant between-group differences were observed in the additive Euroscore, plasma levels of NTproBNP, high-sensitivity troponin-T, creatinine, and estimated glomerular filtration rate (eGFR) as accepted risk factors for postoperative renal dysfunction.

\section{Hemodynamics and metabolism}

Despite a comparable preoperative baseline, MAP after induction of anesthesia was significantly lower in the BIC group. Comparably, mean PAP was lower in these patients upon arrival on the ICU. No further significant between-group differences in hemodynamics were observed (Table 4). Maximal postoperative blood glucose and lactate levels, as well as maximal $\mathrm{Ph}$, were higher in the BIC group (Table 5).

\section{Intra- and postoperative treatments}

While no significant differences in the need for mechanical circulatory support were observed (Table 2), 
Table 1 Demographic data and preoperative risk stratification

\begin{tabular}{|c|c|c|c|c|}
\hline & Total cohort & $\begin{array}{l}\text { Control - no } \\
\text { bicarbonate }\end{array}$ & $\begin{array}{l}\text { Intervention - } \\
\text { bicarbonate }\end{array}$ & $\begin{array}{l}\text { Significance, } \\
P \text {-value }\end{array}$ \\
\hline Number & 584 & $304(52.1 \%)$ & $280(47.9 \%)$ & \\
\hline \multicolumn{5}{|l|}{ Demographics } \\
\hline Age, years & $68.5(67,69)$ & $68.5(67,70)$ & $68.5(67,70)$ & 0.675 \\
\hline Male & $381(65.2 \%)$ & $201(66.1 \%)$ & $180(64.3 \%)$ & 0.706 \\
\hline Female & $203(34.8 \%)$ & $103(33.9 \%)$ & $100(35.7 \%)$ & \\
\hline Height, cm & $\begin{array}{l}171(170 \\
172)\end{array}$ & $171(170,173)$ & $171(170,172)$ & 0.741 \\
\hline Weight, Kg & $\begin{array}{l}81.3(79.9 \\
82.6)\end{array}$ & $82.3(80.6,84.1)$ & $80.1(78.1,82.1)$ & 0.103 \\
\hline BMI $\left(\mathrm{Kg} / \mathrm{m}^{2}\right)$ & $\begin{array}{l}27.2(26.8 \\
27.7)\end{array}$ & $27.4(26.8,27.9)$ & $26.9(26.5,27.8)$ & 0.098 \\
\hline \multicolumn{5}{|l|}{ Cardiovascular risk factors } \\
\hline Diabetes mellitus & $158(27.1 \%)$ & $72(23.8 \%)$ & $86(30.7 \%)$ & 0.073 \\
\hline Arterial hypertension & $484(82.9 \%)$ & $250(82.2 \%)$ & $234(83.6 \%)$ & 0.751 \\
\hline Smoking (former or present smoker) & $246(42.3 \%)$ & $125(41.5 \%)$ & $121(43.2 \%)$ & 0.744 \\
\hline Hyperlipidemia & $410(70.2 \%)$ & $217(71.4 \%)$ & $193(68.9 \%)$ & 0.578 \\
\hline Arterial vascular disease & $135(23.1 \%)$ & $62(20.4 \%)$ & $73(26.1 \%)$ & 0.127 \\
\hline PAH (systolic > $60 \mathrm{mmHg})(\mathrm{n}=223)$ & $32(14.3 \%)$ & $12(11.1 \%)$ & $20(17.4 \%)$ & 0.252 \\
\hline PAH (systolic > $40 \mathrm{mmHg})(\mathrm{n}=223)$ & $112(50.2 \%)$ & $56(51.9 \%)$ & $56(48.7 \%)$ & 0.736 \\
\hline NYHA I/II & $348(59.6 \%)$ & $184(60.5 \%)$ & $164(58.6 \%)$ & 0.692 \\
\hline NYHA III/IV & $236(40.4 \%)$ & $120(39.5 \%)$ & $116(41.4 \%)$ & \\
\hline LVEF $<30 \%$ & $32(5.5 \%)$ & $18(6.0 \%)$ & $14(5.0 \%)$ & 0.658 \\
\hline LVEF 30 to $50 \%$ & $139(24.0 \%)$ & $68(22.6 \%)$ & $71(25.5 \%)$ & \\
\hline LVEF > 50\% & $408(70.5 \%)$ & $215(71.4 \%)$ & $193(69.4 \%)$ & \\
\hline ASA & $3(3,3)$ & $3(3,3)$ & $3(3,3)$ & 0.801 \\
\hline Add. EuroSCORE & $5(5,5)$ & $5(5,5)$ & $4(5,5)$ & 0.455 \\
\hline Add. EuroSCORE 0 to 2 & $134(22.9 \%)$ & $63(20.7 \%)$ & $71(25.4 \%)$ & 0.300 \\
\hline Add. EurOSCORE 3 to 5 & $205(35.1 \%)$ & $114(37.5 \%)$ & 91 (32.5\%) & \\
\hline Add. EuroSCORE $>5$ & $245(42.0 \%)$ & $127(41.8 \%)$ & $118(42.1 \%)$ & \\
\hline \multicolumn{5}{|l|}{ Other risk factors } \\
\hline Pulmonary disease & $91(15.6 \%)$ & $46(15.1 \%)$ & $45(16.1 \%)$ & 0.843 \\
\hline Neurological disease & $70(12.0 \%)$ & $36(11.8 \%)$ & $34(12.1 \%)$ & 0.988 \\
\hline Left main stem disease & $84(14.4 \%)$ & $42(13.8 \%)$ & $42(15.0 \%)$ & 0.772 \\
\hline Previous cardiac or thoracic vascular surgery & $63(10.8 \%)$ & $31(10.2 \%)$ & $32(11.4 \%)$ & 0.730 \\
\hline History of myocardial infarction & $132(22.6 \%)$ & $80(26.3 \%)$ & $52(18.6 \%)$ & 0.033 \\
\hline Troponin-T positive status & $14(2.4 \%)$ & $11(3.6 \%)$ & $3(1.1 \%)$ & 0.082 \\
\hline $\begin{array}{l}\text { History of cardiogenic shock or acute decompensated } \\
\text { heart failure }\end{array}$ & $71(12.2 \%)$ & $32(10.6 \%)$ & $39(13.9 \%)$ & 0.278 \\
\hline \multicolumn{5}{|l|}{ Preoperative physiological profile } \\
\hline MAP, $\mathrm{mmHg}$ & $97(96,98)$ & $98(96,100)$ & $96(93,99)$ & 0.305 \\
\hline Heart rate, bpm & $68(67,70)$ & $68.5(67,70)$ & $68(67,70)$ & 0.918 \\
\hline Mean $\mathrm{ScO} 2_{\text {room }} \%$ & $63(62,63)$ & $62(62,64)$ & $63(62,64)$ & 0.695 \\
\hline Mean $\mathrm{ScO}_{\mathrm{ox},} \%$ & $68(67,68.17)$ & $68(67,69)$ & $68(67,69)$ & 0.926 \\
\hline $\mathrm{ScO} 2_{\operatorname{minox},} \%$ & $66(65,67)$ & $66(65,67)$ & $65(65,67)$ & 0.924 \\
\hline NTproBNP, pg/ml $(n=389)$ & $\begin{array}{l}455(355 \\
519)\end{array}$ & $392(312,496)$ & $517(391,686)$ & 0.234 \\
\hline hsTNT, pg/ml $(n=389)$ & $9(8,11)$ & $9(8,13)$ & $9(7,11)$ & 0.850 \\
\hline
\end{tabular}

Demographics, risk factors, and preoperative physiological status are shown for patients undergoing elective, on-pump cardiac surgery with or without a perioperative infusion of bicarbonate. BMI: body mass index; PAH: pulmonary arterial hypertension; NYHA: New York Heart Association classification; LVEF: left ventricular ejection fraction; ASA: American Society of Anesthesiology classification; Add.: additive; MAP: mean arterial pressure. ScO2: cerebral oxygen saturation determined bi-hemispherically by near-infrared spectroscopy; $\mathrm{ScO}_{\text {room: }}$ mean of both hemispheres determined when breathing room air; $\mathrm{ScO} 2$ ox: mean of both hemispheres determined during application of oxygen-enriched air. $\mathrm{ScO}_{\text {minox }}$ : minimal $\mathrm{ScO} 2$ from the left or right hemisphere determined during application of oxygen-enriched air; NTproBNP: N-terminal pro B-type natriuretic peptide; hsTNT: high-sensitivity troponin-T. Data are given as absolute numbers (percentage) for nominal variables and median (95\% confidence interval). 
Table 2 Surgical procedures and performance

\begin{tabular}{|c|c|c|c|c|}
\hline & Total cohort & Control - no bicarbonate & Intervention - bicarbonate & Significance, $P$-value \\
\hline Number & 584 & $304(52.1 \%)$ & $280(47.9 \%)$ & \\
\hline \multicolumn{5}{|l|}{ Type of procedure } \\
\hline CABG & $222(38.1)$ & $110(36.3 \%)$ & $112(40.0 \%)$ & 0.160 \\
\hline Valve surgery +/- varia & $176(30.1 \%)$ & $93(30.7 \%)$ & $82(28.9 \%)$ & \\
\hline CABG +/- valve +/- varia & $120(20.6 \%)$ & $71(23.4 \%)$ & $49(17.5 \%)$ & \\
\hline Aortic surgery +/- valve +/- varia & $54(9.3 \%)$ & $21(6.9 \%)$ & $33(11.8 \%)$ & \\
\hline Various procedures & $10(1.7 \%)$ & $8(2.6 \%)$ & $3(1.1 \%)$ & \\
\hline Aortic surgery +/- CABG & $2(0.3 \%)$ & $1(0.3 \%)$ & $1(0.4 \%)$ & \\
\hline Duration of surgery, minutes & $254(247,261)$ & $249(242,260)$ & $258(250,268)$ & 0.406 \\
\hline Duration of CPB, minutes & $115(110,119)$ & $1159(107,122)$ & $115(109,120)$ & 0.756 \\
\hline Crossclamp time, minutes & $89.5(84,93)$ & $88.5(82,93)$ & $90(83,96)$ & 0.539 \\
\hline ECLS & $7(1.2 \%)$ & $3(1.0 \%)$ & $4(1.4 \%)$ & 0.913 \\
\hline \multicolumn{5}{|l|}{ IABP } \\
\hline No IABP & $556(95.2 \%)$ & $289(95.1 \%)$ & 267 (95.4\%) & 0.445 \\
\hline IABP before CPB & $6(1.0 \%)$ & $2(0.7 \%)$ & $4(1.4 \%)$ & \\
\hline IABP during CPB & $8(1.4 \%)$ & $6(2.0 \%)$ & $2(0.7 \%)$ & \\
\hline IABP after CPB & $8(1.4 \%)$ & $3(1.0 \%)$ & $5(1.8 \%)$ & \\
\hline IABP on ICU & $6(1.0 \%)$ & $4(1.3 \%)$ & $2(0.7 \%)$ & \\
\hline Circulatory arrest & $21(3.6 \%)$ & $13(4.3 \%)$ & $8(2.9 \%)$ & 0.485 \\
\hline Lowest body temperature, ${ }^{\circ} \mathrm{C}$ & $32(32,32)$ & $32(32,32)$ & $32(32,32)$ & 0.615 \\
\hline
\end{tabular}

Surgical procedures and surgical core data are shown for patients undergoing elective on-pump cardiac surgery with or without a perioperative infusion of bicarbonate. CABG: coronary artery bypass grafting; CPB: cardiopulmonary bypass; ECLS: extracorporeal life support; IABP: intra-aortic balloon pump. Data are given as absolute numbers (percentage) for nominal variables and as median ( $95 \%$ confidence interval).

Table 3 Preoperative, intraoperative and postoperative therapies

\begin{tabular}{|c|c|c|c|c|}
\hline & Total cohort & Control - no bicarbonate & Intervention - bicarbonate & Significance, $P$-value \\
\hline Number & 584 & $304(52.1 \%)$ & $280(47.9 \%)$ & \\
\hline \multicolumn{5}{|l|}{ Preoperative treatments } \\
\hline Beta-blocking agents & $442(75.8 \%)$ & $234(77.0 \%)$ & $208(74.6 \%)$ & 0.558 \\
\hline ACE-inhibitors/ARB & $424(72.7 \%)$ & $225(74.0 \%)$ & $199(71.3 \%)$ & 0.526 \\
\hline Diuretics & $328(56.4 \%)$ & $173(56.9 \%)$ & $155(55.8 \%)$ & 0.844 \\
\hline Vasodilators & $209(35.8 \%)$ & $110(36.2 \%)$ & 99 (35.5\%) & 0.929 \\
\hline Phenprocoumon & $11(1.9 \%)$ & $4(1.3 \%)$ & $7(2.5 \%)$ & 0.451 \\
\hline Aspirine & $373(64.1 \%)$ & $203(66.8 \%)$ & $170(61.2 \%)$ & 0.185 \\
\hline Clopidogrel & $53(9.1 \%)$ & $24(7.9 \%)$ & $29(10.4 \%)$ & 0.366 \\
\hline Heparin, intravenous & $95(16.3 \%)$ & $62(20.4 \%)$ & $33(11.8 \%)$ & 0.007 \\
\hline Lipid-lowering drugs & $356(61.1 \%)$ & $197(64.8 \%)$ & $159(57.0 \%)$ & 0.065 \\
\hline Insulin & $64(11.0 \%)$ & $30(9.9 \%)$ & $34(12.2 \%)$ & 0.446 \\
\hline Antibiotics & $64(11.0 \%)$ & $33(10.9 \%)$ & $31(11.1 \%)$ & 0.973 \\
\hline Nitrates, intravenous & $29(5.0 \%)$ & $17(5.6 \%)$ & $12(4.3 \%)$ & 0.599 \\
\hline Levosimendan & $16(2.7 \%)$ & $7(2.3 \%)$ & $9(3.2 \%)$ & 0.669 \\
\hline \multicolumn{5}{|l|}{ Intraoperative fluids } \\
\hline Cristalline fluids, ml & $1,948(1,903,1,993)$ & $1,982(, 1921,2,042)$ & $1,911(1,844,1,978)$ & 0.120 \\
\hline Colloidal fluids, ml & $800(756,843)$ & $791(730,852)$ & $809(748,870)$ & 0.680 \\
\hline \multicolumn{5}{|c|}{ Postoperative fluids within $24 \mathrm{~h}$} \\
\hline Cristalline fluids, ml & $2,719(2,667,2,771)$ & $2,590(2,519,2661)$ & $2,859(2,786,2,933)$ & $<0.0001$ \\
\hline Colloidal fluids, ml & $1,476(1,412,1,541)$ & $1,404(1,314,1,494)$ & $1,555(1,462,1,647)$ & 0.022 \\
\hline \multicolumn{5}{|c|}{ Intraoperative blood transfusion } \\
\hline PRC, patients transfused & $314(53.8 \%)$ & $166(54.6 \%)$ & $148(52.9 \%)$ & 0.734 \\
\hline units transfused & $1(0,1)$ & $1(0,1)$ & $1(0,1)$ & 0.925 \\
\hline FFP, patients transfused & $38(6.5 \%)$ & $20(6.6 \%)$ & $18(6.4 \%)$ & 0.925 \\
\hline
\end{tabular}


Table 3 Preoperative, intraoperative and postoperative therapies (Continued)

\begin{tabular}{|c|c|c|c|c|}
\hline units transfused & $0[0-0]$ & $0[0-0]$ & $0[0-0]$ & 0.871 \\
\hline TC, patients transfused & $30(5.1 \%)$ & $16(5.3 \%)$ & $14(5.0 \%)$ & 0.965 \\
\hline units transfused & $0(0,0)$ & $0(0,0)$ & $0(0,0)$ & 0.980 \\
\hline Cellsaver, number patients & $22(3.8 \%)$ & $15(4.9 \%)$ & $7(2.5 \%)$ & 0.185 \\
\hline \multicolumn{5}{|c|}{ Postoperative blood transfusion } \\
\hline PRC, patients transfused & $253(43.3 \%)$ & $129(42.4 \%)$ & $124(44.3 \%)$ & 0.713 \\
\hline units transfused & $0.00(0.00,0.00)$ & $0.00(0.00,0.00)$ & $0.00(0.00,0.88)$ & 0.505 \\
\hline FFP, patients transfused & $43(7.4 \%)$ & $22(7.2 \%)$ & $21(7.5 \%)$ & 0.971 \\
\hline units transfused & $0.00(0.00,0.00)$ & $0.00(0.00,0.00)$ & $0.00(0.00,0.00)$ & 0.907 \\
\hline TC, patients transfused & $43(7.4 \%)$ & $22(7.2 \%)$ & $21(7.5 \%)$ & 0.980 \\
\hline units transfused & $0.00(0.00,0.00)$ & $0.00(0.00,0.00)$ & $0.00(0.00,0.00)$ & 0.881 \\
\hline \multicolumn{5}{|c|}{ Intraoperative vasoactive and inotropic treatments } \\
\hline Levosimendan, n (\%) & $24(4.1 \%)$ & $11(3.6 \%)$ & $13(4.6 \%)$ & 0.679 \\
\hline Before CPB & $23(3.9 \%)$ & $11(3.6 \%)$ & $12(4.3 \%)$ & 0.531 \\
\hline After CPB & $1(0.2 \%)$ & $0(0.0 \%)$ & $1(0.4 \%)$ & \\
\hline Noradrenaline, n (\%) & $522(89.4 \%)$ & $268(88.2 \%)$ & $254(90.7 \%)$ & 0.386 \\
\hline$<0.3 \mathrm{mg} / \mathrm{h}$ & $261(44.7 \%)$ & $148(48.7 \%)$ & $113(40.4 \%)$ & 0.069 \\
\hline 0.3 to $0.6 \mathrm{mg} / \mathrm{h}$ & $187(32.0 \%)$ & $87(28.6 \%)$ & $100(35.7 \%)$ & \\
\hline$>0.6 \mathrm{mg} / \mathrm{h}$ & $74(12.7 \%)$ & $33(10.9 \%)$ & $41(14.6 \%)$ & \\
\hline Vasopressin, n (\%) & $49(8.4 \%)$ & $19(6.2 \%)$ & $30(10.7 \%)$ & 0.073 \\
\hline$<3 \mathrm{U} / \mathrm{h}$ & $33(5.7 \%)$ & $11(3.6 \%)$ & $22(7.9 \%)$ & 0.109 \\
\hline 3 to $6 \mathrm{U} / \mathrm{h}$ & $15(2.6 \%)$ & $8(2.6 \%)$ & $7(2.5 \%)$ & \\
\hline$>6 \mathrm{U} / \mathrm{h}$ & $1(0.2 \%)$ & $0(0.0 \%)$ & $1(0.4 \%)$ & \\
\hline Dobutamin, n (\%) & $330(56.5 \%)$ & $178(58.6 \%)$ & $152(54.3 \%)$ & 0.339 \\
\hline$<15 \mathrm{mg} / \mathrm{h}$ & $130(22.3 \%)$ & $70(23.0 \%)$ & $60(21.4 \%)$ & 0.278 \\
\hline 15 to $30 \mathrm{mg} / \mathrm{h}$ & $170(29.1 \%)$ & $96(31.6 \%)$ & $74(26.4 \%)$ & \\
\hline$>30 \mathrm{mg} / \mathrm{h}$ & $30(5.1 \%)$ & $12(3.9 \%)$ & $18(6.4 \%)$ & \\
\hline PDE-III inhibitors, n (\%) & $249(42.6 \%)$ & $121(39.8 \%)$ & $128(45.7 \%)$ & 0.174 \\
\hline Low dose & $35(6.0 \%)$ & $14(4.6 \%)$ & $21(7.5 \%)$ & 0.126 \\
\hline Moderate dose & $198(33.9 \%)$ & $96(31.6 \%)$ & $102(36.4 \%)$ & \\
\hline High dose & $16(2.7 \%)$ & $11(3.6 \%)$ & $5(1.8 \%)$ & \\
\hline \multicolumn{5}{|c|}{ Postoperative vasoactive and inotropic treatment, n (\%) } \\
\hline Levosimendan & $24(4.1 \%)$ & $12(3.9 \%)$ & $12(4.3 \%)$ & 0.998 \\
\hline Noradrenalin & $494(84.6 \%)$ & $246(80.9 \%)$ & $248(88.6 \%)$ & 0.015 \\
\hline$<0.3 \mathrm{mg} / \mathrm{h}$ & $262(44.9 \%)$ & $147(48.4 \%)$ & $115(41.1 \%)$ & 0.0013 \\
\hline 0.3 to $0.6 \mathrm{mg} / \mathrm{h}$ & $140(24.0 \%)$ & $58(19.1 \%)$ & $82(29.3 \%)$ & \\
\hline$>0.6 \mathrm{mg} / \mathrm{h}$ & $92(15.8 \%)$ & $41(13.5 \%)$ & $51(18.2 \%)$ & \\
\hline Vasopressin & $64(11.0 \%)$ & $29(9.5 \%)$ & $35(12.5 \%)$ & 0.312 \\
\hline$<3 \mathrm{U} / \mathrm{h}$ & 49 (8.4\%) & $19(6.2 \%)$ & $30(10.7 \%)$ & 0.116 \\
\hline 3 to 6 Uh & $13(2.2 \%)$ & $8(2.6 \%)$ & $5(1.8 \%)$ & \\
\hline$>6 \mathrm{U} / \mathrm{h}$ & $2(0.3 \%)$ & $2(0.7 \%)$ & $0(0.0 \%)$ & \\
\hline Dobutamin & $386(66.1 \%)$ & $207(68.1 \%)$ & 179 (63.9\%) & 0.330 \\
\hline$<15 \mathrm{mg} / \mathrm{h}$ & $184(31.5 \%)$ & 97 (31.9\%) & 87 (31.1\%) & 0.728 \\
\hline 15 to $30 \mathrm{mg} / \mathrm{h}$ & $164(28.1 \%)$ & $90(29.6 \%)$ & $74(26.4 \%)$ & \\
\hline$>30 \mathrm{mg} / \mathrm{h}$ & $38(6.5 \%)$ & $20(6.6 \%)$ & $18(6.4 \%)$ & \\
\hline PDE III inhibitors & $312(53.5 \%)$ & $156(51.3 \%)$ & 156 (55.9\%) & 0.304 \\
\hline Low dose & $60(10.3 \%)$ & $32(10.5 \%)$ & $28(10.0 \%)$ & 0.665 \\
\hline Moderate dose & $224(38.4 \%)$ & $110(36.2 \%)$ & 114 (40.9\%) & \\
\hline High dose & $28(4.8 \%)$ & $14(4.6 \%)$ & $14(5.0 \%)$ & \\
\hline
\end{tabular}

Perioperative pharmacological treatments are shown for patients undergoing elective on-pump cardiac surgery with or without a perioperative infusion of bicarbonate. ACE-inhibitors: angiotensin converting enzyme inhibitors; ARB: angiotensin receptor blocker; PRC: packed red cells; FFP: fresh frozen plasma; TC: thrombocyte concentrate; PDE-III inhibitors; phosphodiesterase III inhibitors. Data are given as absolute numbers (percentage) for nominal variables, and as median ( $95 \%$ confidence interval) for non-normally distributed data, and mean (95\% confidence interval) for normally distributed variables. 
Table 4 Intraoperative and postoperative hemodynamics

\begin{tabular}{|c|c|c|c|c|}
\hline & Total cohort & Control - no bicarbonate & Intervention - bicarbonate & Significance, $P$-value \\
\hline Number of patients & 584 & 304 & 280 & \\
\hline HR, bpm, preoperative & $68(67,70)$ & $68.5(67,70)$ & $68(67,70)$ & 0.875 \\
\hline MAP, mmHg, preoperative & $96(95,98)$ & $97(95,100)$ & $95(93,97)$ & 0.396 \\
\hline MAP, mmHg, before induction & $85(82,85)$ & $87(85,90)$ & $80(76,83)$ & $<0.0001$ \\
\hline MAP, $\mathrm{mmHg}, 30$ minutes before CPB & $70(70,71)$ & $70(70,72)$ & $70(68,71)$ & 0.135 \\
\hline MAP, mmHg, 30 minutes after CPB & $69(68,70)$ & $70(69,71)$ & $69(67,70)$ & 0.109 \\
\hline MAP, $\mathrm{mmHg}$, end of surgery & $72(71,73)$ & $73(71,74)$ & $71(70,73)$ & 0.150 \\
\hline MAP, mmHg, ICU admission & $76(75,77)$ & $76(75,78)$ & $75(73,78)$ & 0.339 \\
\hline MAP, $\mathrm{mmHg}, 2 \mathrm{~h}$ after ICU admission & $73(72,74)$ & $73(71,75)$ & $73(72,74)$ & 0.833 \\
\hline MAP, $\mathrm{mmHg}, 4 \mathrm{~h}$ after ICU admission & $74(72,75)$ & $74(72,76)$ & $73(72,74)$ & 0.541 \\
\hline MAP, $\mathrm{mmHg}, 6 \mathrm{~h}$ after ICU admission & $73(73,74)$ & $74(73,75)$ & $73(72,75)$ & 0.361 \\
\hline MAP, $\mathrm{mmHg}, 8 \mathrm{~h}$ after ICU admission & $74(73,75)$ & $74(72,75)$ & $74(72,75)$ & 0.632 \\
\hline CVP, mmHg, after induction & $11(10,11)$ & $11(10,11)$ & $11(10,12)$ & 0.340 \\
\hline CVP, mmHg, 30 minutes before CPB & $8(8,8)$ & $8(8,9)$ & $8(7,9)$ & 0.561 \\
\hline CVP, mmHg, 30 minutes after CPB & $11(10,11)$ & $10(10,11)$ & $11(10,12)$ & 0.663 \\
\hline $\mathrm{CVP}, \mathrm{mmHg}$, end of surgery & $12(11,12)$ & $12(11,12)$ & $12(11,12)$ & 0.396 \\
\hline CVP, mmHg, ICU admission & $15(15,16)$ & $16(15,16)$ & $15(15,16)$ & 0.689 \\
\hline CVP, $\mathrm{mmHg}, 2 \mathrm{~h}$ after ICU admission & $15(15,16)$ & $16(15,16)$ & $15(14,15)$ & 0.129 \\
\hline CVP, $\mathrm{mmHg}, 4 \mathrm{~h}$ after ICU admission & $15(15,16)$ & $15(14,16)$ & $15(15,16)$ & 0.425 \\
\hline CVP, $\mathrm{mmHg}, 6 \mathrm{~h}$ after ICU admission & $14(14,15)$ & $14(14,15)$ & $14(14,15)$ & 0.358 \\
\hline CVP, $\mathrm{mmHg}, 8 \mathrm{~h}$ after ICU admission & $13(13,14)$ & $13(12,14)$ & $13(12,14)$ & 0.801 \\
\hline MPAP, mmHg, After induction & $25(24,27)$ & $26(24,29) n=61$ & $25(22,29) n=46$ & 0.380 \\
\hline MPAP, mmHg, 30 minutes after CPB & $25(23,27)$ & $26(23,28) n=76$ & $25(22,27) n=75$ & 0.466 \\
\hline MPAP, mmHg, 30 minutes after CPB & $26(24,27)$ & $25(23,28) n=104$ & $26(24,28) n=97$ & 0.324 \\
\hline MPAP, mmHg, end of surgery & $26(25,28)$ & $26(24,28) n=107$ & $27(24,29) n=94$ & 0.659 \\
\hline MPAP, mmHg, ICU admission & $29(28,30)$ & $30(29,33) n=113$ & $27(26,29) n=84$ & 0.006 \\
\hline MPAP, $\mathrm{mmHg}, 2 \mathrm{~h}$ after ICU admission & $30(29,31)$ & $30(29,31) n=120$ & $29(28,31) n=104$ & 0.538 \\
\hline MPAP, $\mathrm{mmHg}, 4 \mathrm{~h}$ after ICU admission & $30(29,31)$ & $31(29,32) n=126$ & $29(28,30) n=107$ & 0.448 \\
\hline MPAP, $\mathrm{mmHg}, 6 \mathrm{~h}$ after ICU admission & $29(27,29)$ & $29(27,30) n=125$ & $28(27,30) n=109$ & 0.892 \\
\hline MPAP, $\mathrm{mmHg}, 8 \mathrm{~h}$ after ICU admission & $28(27,29)$ & $28(26,30) n=128$ & $28(26,30) n=113$ & 0.731 \\
\hline $\mathrm{Cl}, \mathrm{L} / \mathrm{min} / \mathrm{m} 2$, after induction & $2.45(2.24,2.66)$ & $2.46(2.14,2.77) n=56$ & $2.44(2.17,2.71) n=38$ & 0.947 \\
\hline $\mathrm{Cl}, \mathrm{L} / \mathrm{min} / \mathrm{m} 2,30$ minutes before CPB & $2.68(2.57,2.80)$ & $2.62(2.45,2.79) n=76$ & $2.75(2.58,2.92) n=75$ & 0.269 \\
\hline $\mathrm{Cl}, \mathrm{L} / \mathrm{min} / \mathrm{m} 2,30$ minutes after CPB & $3.22(3.08,3.37)$ & $3.14(2.94,3.34) n=104$ & $3.31(3.09,3.53) n=97$ & 0.261 \\
\hline $\mathrm{Cl}, \mathrm{L} / \mathrm{min} / \mathrm{m} 2$, end of surgery & $3.02(2.92-3.13)$ & $2.95(2.79,3.11) n=107$ & $3.11(2.97,3.25) n=94$ & 0.140 \\
\hline $\mathrm{Cl}, \mathrm{L} / \mathrm{min} / \mathrm{m} 2, \mathrm{ICU}$ admission & $2.80(2.69,2.92)$ & $2.87(2.73,3.01) n=113$ & $2.71(2.52,2.89) n=84$ & 0.171 \\
\hline $\mathrm{Cl}, \mathrm{L} / \mathrm{min} / \mathrm{m} 2,2 \mathrm{~h}$ after ICU admission & $3.06(2.95,3.17)$ & $3.07(2.92,3.22) n=120$ & $3.05(2.88,3.21) n=104$ & 0.835 \\
\hline $\mathrm{Cl}, \mathrm{L} / \mathrm{min} / \mathrm{m} 2,4 \mathrm{~h}$ after ICU admission & $3.12(3.02,3.23)$ & $3.10(2.98,3.23) n=126$ & $3.15(2.98,3.32) n=107$ & 0.675 \\
\hline $\mathrm{Cl}, \mathrm{L} / \mathrm{min} / \mathrm{m} 2,6 \mathrm{~h}$ after ICU admission & $3.24(3.14,-3.35)$ & $3.22(3.10,3.34) n=125$ & $3.27(3.08,3.46) n=109$ & 0.686 \\
\hline $\mathrm{Cl}, \mathrm{L} / \mathrm{min} / \mathrm{m} 2,8 \mathrm{~h}$ after ICU admission & $3.18(3.09,3.28)$ & $3.23(3.11,3.35) n=128$ & $3.13(2.98,3.28) n=113$ & 0.301 \\
\hline $\mathrm{SvO}_{2}, \%$, after induction $(\mathrm{n}=118)$ & $79(77,80)$ & $79(78,81) n=61$ & $77(75,79) n=46$ & 0.060 \\
\hline $\mathrm{SvO}_{2}, \%, 30$ minutes before $\mathrm{CPB}(\mathrm{n}=147)$ & $82(81,82)$ & $82(81,83) n=76$ & $81(80,82) n=75$ & 0.357 \\
\hline $\mathrm{SvO}_{2}, \%, 30$ minutes after CPB $(n=177)$ & $78(77,79)$ & $79(77,80) n=104$ & $78(77,80) n=97$ & 0.735 \\
\hline $\mathrm{SvO}_{2}, \%$, end of surgery $(n=179)$ & $77(76,78)$ & $77(76,79) n=107$ & $77(76,79) n=94$ & 0.988 \\
\hline $\mathrm{SvO}_{2}, \%, \mathrm{ICU}$ admission $(\mathrm{n}=192)$ & $71(70,72)$ & $71(70,73) n=113$ & $70(68,72) n=84$ & 0.380 \\
\hline $\mathrm{SvO}_{2}, \%, 2 \mathrm{~h}$ after ICU admission $(\mathrm{n}=287)$ & $69(68,70)$ & $69(67,70) n=120$ & $69(67,70) n=104$ & 0.931 \\
\hline $\mathrm{SvO}_{2}, \% 4 \mathrm{~h}$ after ICU admission $(\mathrm{n}=309)$ & $68(67,69)$ & $67(66,69) n=126$ & $68(67,69) n=107$ & 0.322 \\
\hline $\mathrm{SvO}_{2}, \%, 6 \mathrm{~h}$ after ICU admission $(\mathrm{n}=316)$ & $69(68,70)$ & $69(68,70) n=125$ & $69(67,70) n=109$ & 0.587 \\
\hline $\mathrm{SvO}_{2}, \%, 8 \mathrm{~h}$ after ICU admission & $69(68,70)$ & $69(68,71) n=128$ & $69(67,70) n=113$ & 0.264 \\
\hline
\end{tabular}

Data are given as median/mean (95\% confidence interval for the median/mean) as appropriate. HR: heart rate; MAP mean arterial blood pressure; CVP: central venous pressure; MPAP: mean pulmonary arterial pressure; $\mathrm{Cl}$ : cardiac index; $\mathrm{SvO}_{2}$ : mixed venous oxygen saturation. $\mathrm{MPAP}, \mathrm{Cl}$, and $\mathrm{SvO}{ }_{2}$ were not available in every patient. 
Table 5 Renal function and metabolism

\begin{tabular}{|c|c|c|c|c|}
\hline & Total cohort & $\begin{array}{l}\text { Control - no } \\
\text { bicarbonate }\end{array}$ & $\begin{array}{l}\text { Intervention - } \\
\text { bicarbonate }\end{array}$ & $\begin{array}{l}\text { Significance, } \\
P \text {-value }\end{array}$ \\
\hline Number & 584 & $304(52.1 \%)$ & $280(47.9 \%)$ & \\
\hline \multicolumn{5}{|l|}{ Diuresis and fluid balance } \\
\hline Postoperative diuresis within $24 \mathrm{~h}, \mathrm{ml}$ & $\begin{array}{l}2,844(2,744 \\
2,944)\end{array}$ & $2,723(2,564,2,881)$ & $2,977(2,861,3,092)$ & 0.012 \\
\hline Postoperative cristalloid fluid balance, $\mathrm{ml}$ & $\begin{array}{l}-124(-225 \\
-22)\end{array}$ & $-133(-295,30)$ & $-114(-231,4)$ & 0.855 \\
\hline Postoperative surgical drainage, $\mathrm{ml}$ & $927(887,968)]$ & $933(875,991)$ & $921(865,978)$ & 0.782 \\
\hline Postoperative colloidals - drainage fluid balance, $\mathrm{ml}$ & $549(480,618)$ & $471(374,569)$ & $633(536,731)$ & 0.022 \\
\hline \multicolumn{5}{|l|}{ Postoperative diuretics, number of patients } \\
\hline Furosemide & $280(47.9 \%)$ & $142(46.7 \%)$ & $138(49.3 \%)$ & 0.590 \\
\hline Torasemide & $462(79.1 \%)$ & $242(79.6 \%)$ & $220(78.6 \%)$ & 0.838 \\
\hline $\mathrm{HCT}$ & $200(34.2 \%)$ & $102(33.6 \%)$ & $98(35.0 \%)$ & 0.779 \\
\hline Other diuretics & $34(5.8 \%)$ & $20(6.6 \%)$ & $14(5.0 \%)$ & 0.524 \\
\hline \multicolumn{5}{|l|}{ Renal function } \\
\hline Creatinine baseline, $\mu \mathrm{mol} / \mathrm{L}$ & $81(79,83)$ & $81(78,84)$ & $82(78,84)$ & 0.787 \\
\hline Creatinine maximum*, $\mu \mathrm{mol} / \mathrm{L}$ & $93(90,96)$ & $93(88,97)$ & $93(89,97)$ & 0.785 \\
\hline Creatinine at discharge ${ }^{*}, \mu \mathrm{mol} / \mathrm{L}$ & $82(80,87)$ & $84(80,88)$ & $80(80,87)$ & 0.153 \\
\hline \multicolumn{5}{|l|}{ Maximum relative increase in creatinine* } \\
\hline$<25 \%$ & $369(63.2 \%)$ & $204(67.1 \%)$ & $165(58.9 \%)$ & 0.217 \\
\hline$>25 \%$ & $123(21.1 \%)$ & $57(18.8 \%)$ & $66(23.6 \%)$ & \\
\hline$>50 \%$ & $56(9.6 \%)$ & $25(8.2 \%)$ & $31(11.1 \%)$ & \\
\hline$>100 \%$ & $36(6.2 \%)$ & $18(5.9 \%)$ & $18(6.4 \%)$ & \\
\hline eGFR baseline, $\mathrm{ml} / \mathrm{min}$ & $82(80,84)$ & $81(78,84)$ & $83(80,87)$ & 0.434 \\
\hline eGFR minimum*, $\mathrm{ml} / \mathrm{min}$ & $69(67,71)$ & $69(66,72)$ & $70(66,73)$ & 0.744 \\
\hline eGFR at discharge ${ }^{*}, \mathrm{ml} / \mathrm{min}(\mathrm{n}=566)$ & $82(79,85)$ & $81(77,86)$ & $83(79,86)$ & 0.586 \\
\hline \multicolumn{5}{|c|}{$\begin{array}{l}\text { AKI grading according to creatinine changes (new RRT = grade } \\
\text { 3), number patients }\end{array}$} \\
\hline Grade 1 & $85(14.6 \%)$ & $38(12.5 \%)$ & $47(16.8 \%)$ & 0.478 \\
\hline Grade 2 & $7(1.2 \%)$ & $3(1.0 \%)$ & $4(1.4 \%)$ & \\
\hline Grade 3 & $74(12.7 \%)$ & $39(12.8 \%)$ & $35(12.5 \%)$ & \\
\hline \multicolumn{5}{|c|}{$\begin{array}{l}\text { AKI grading according to diuresis** (new RRT }=\text { grade } 3 \text { ), number } \\
\text { patients }\end{array}$} \\
\hline Grade 1 & $14(2.4 \%)$ & $11(3.6 \%)$ & $3(1.1 \%)$ & 0.135 \\
\hline Grade 2 & $1(0.2 \%)$ & $0(0.0 \%)$ & $1(0.4 \%)$ & \\
\hline Grade 3 & $45(7.7 \%)$ & $21(6.9 \%)$ & $24(8.6 \%)$ & \\
\hline \multicolumn{5}{|l|}{ AKI grading, total**, number patients } \\
\hline Grade 1 & $90(15.4 \%)$ & $44(14.5 \%)$ & $46(16.4 \%)$ & 0.753 \\
\hline Grade 2 & $8(1.4 \%)$ & $3(1.0 \%)$ & $5(1.8 \%)$ & \\
\hline Grade 3 & $75(12.8 \%)$ & $39(12.8 \%)$ & $36(12.9 \%)$ & \\
\hline \multicolumn{5}{|l|}{ New RRT } \\
\hline Patients (\%) & $45(7.7 \%)$ & $22(7.2 \%)$ & $23(8.2 \%)$ & 0.774 \\
\hline Duration of treatment, $\mathrm{h}$ & $144(114,196)$ & $153(110,196)$ & $125(76,227)$ & 0.892 \\
\hline \multicolumn{5}{|l|}{ Intraoperative metabolism } \\
\hline Blood glucose max, mg/dl & $193(189,202)$ & $189(184,193)$ & $197(193,204)$ & 0.005 \\
\hline Blood glucose min, mg/dl & $92(91,93)$ & $91.5(89,94)$ & $92(91,94)$ & 0.181 \\
\hline $\mathrm{pH} \max$ & $\begin{array}{l}7.45(7.44 \\
7.45)\end{array}$ & $7.45(7.45,7.45)$ & $7.45(7.44,7.45)$ & 0.034 \\
\hline $\mathrm{pH}$ min & $\begin{array}{l}7.32(7.32 \\
7.32)\end{array}$ & $7.32(7.31,7.32)$ & $7.32(7.32,7.33)$ & 0.157 \\
\hline Lactate max, mmol/L & $1.7(1.6,1.8)$ & $1.6(1.5,1.7)$ & $1.8(1.6,1.9)$ & 0.005 \\
\hline Lactate min, mmol/L & $0.6(0.6,0.6)$ & $0.6(0.6,0.7)$ & $0.7(0.6,0.7)$ & 0.594 \\
\hline
\end{tabular}


Table 5 Renal function and metabolism (Continued)

\begin{tabular}{|c|c|c|c|c|}
\hline \multicolumn{5}{|l|}{ Postoperative metabolism } \\
\hline Blood glucose max, mg/dl & $\begin{array}{l}169.0(166.0 \\
172.0)\end{array}$ & $163.5(161.0,167.0)$ & $173.5(170.12,177.88)$ & 0.0015 \\
\hline Blood glucose min, mg/dl & $\begin{array}{l}90.09 \\
(88.68,1.51)\end{array}$ & $89.70(87.73,91.67)$ & $90.53(88.48,92.57)$ & 0.566 \\
\hline $\mathrm{pH} \max$ & $\begin{array}{l}7.46(7.46 \\
7.47]\end{array}$ & $7.46(7.45,7.46)$ & $7.47(7.46,7.48)$ & 0.001 \\
\hline $\mathrm{pH} \min$ & $\begin{array}{l}7.32(7.31 \\
7.32)\end{array}$ & $7.32(7.31,7.32)$ & $7.32(7.31,7.33)$ & 0.165 \\
\hline Lactate max, mmol/L & $\begin{array}{l}2.00(1.90, \\
2.10)\end{array}$ & $1.90(1.70,2.00)$ & $2.10(2.00,2.20)$ & 0.010 \\
\hline
\end{tabular}

Fluid balance, diuretics, metabolism, and renal function are shown for patients undergoing elective on-pump cardiac surgery with or without a perioperative infusion of bicarbonate. HCT: hydrochlorothiazide; eGFR: estimated glomerular filtration rate: modifications of diet in renal disease formula; AKI: acute kidney injury; CVVH: continuous-veno-venous hemofiltration; max: maximal; min: minimal. Data are given as absolute numbers (percentage) for nominal variables, median ( $95 \%$ confidence interval) for non-normally distributed data, and mean ( $95 \%$ confidence interval) for normally distributed variables. ${ }^{*}$ Changes in plasma creatinine levels and derived variables were calculated after exclusion of patients treated with renal replacement therapy. ${ }^{* *} \mathrm{AKI}$ grading was performed according to Acute Kidney Injury Network recommendations (9) on the basis of changes in plasma creatinine, need for new renal replacement therapy and time course of diuresis (within $48 \mathrm{~h}$ after surgery).

hemodynamic treatments differed markedly between the groups. Patients in the BIC group were treated with more crystalline and colloidal fluids, and more frequently and with higher doses of noradrenaline (Table 3). No differences were observed in the use of inotropic drugs. No differences in the rate of transfusion of packed red cells, fresh frozen plasma, or thrombocyte concentrates were observed.

\section{Renal outcomes}

Patients in the BIC group had a higher diuresis within the first $24 \mathrm{~h}$ after surgery. No differences were observed in the use of diuretics (Table 5). Renal replacement therapy (RRT) was initiated in $7.2 \%$ of patients in the $\mathrm{CON}$ and $8.2 \%$ of patients in the BIC group. Time to initiation of RRT after ICU admission was 29.0 (19.0 to 39.0$) \mathrm{h}$ in the CON and 35.5 (24.5 to 49.0$) \mathrm{h}$ in the BIC group ( $P$ not significant). The specific changes in plasma creatinine, eGFR and the grading according to the Acute Kidney Injury Network criteria are depicted in Table 5.

\section{General clinical outcomes}

Despite comparable postoperative ventilation times, duration of treatment in the ICU and in the high dependency unit (HDU) were significantly prolonged in the BIC group (Table 6). No differences in direct or indirect measures of morbidity and mortality were observed (Table 6).

\section{Discussion}

AKI is not only a frequent complication in cardiac surgical patients [1] but has also been shown to be independently associated with morbidity and mortality $[10,11]$. Unfortunately, little progress has been made within the last years in the development of strategies to reduce the incidence and improve the prognosis of this complication.

Recently, Haase and coworkers have elegantly delineated a pathophysiological line of evidence that the severity of the renal insult induced by on-pump cardiac surgery may, at least in part, be related to the degree of hemoglobinuria: the histological features of CSA-AKI resemble the pigment nephropathy typically observed during rhabdomyolysis [5]. Since alkalization of the urine is among the established measures to treat rhabdomyolysis [12] they used this concept successfully as a strategy for the prevention of CSA-AKI in a small pilot trial [7]. With respect to these promising findings, the relatively high incidence of CSA-AKI at our institution, the lack of other available measures for preventing renal dysfunction during cardiac surgery [13], and the fact that urine alkalization for the treatment of rhabdomyolysis has a longstanding tradition in clinical medicine [12], we chose to implement this concept into our clinical routine. It is of note that an interdisciplinary working group on this topic also gave a positive recommendation to use hydration and bicarbonate to reduce the nephrotoxic effects of myo- and hemoglobinuria [14].

In contrast to these promising findings, the results of the present prospective observational cohort study show that, in a heterogeneous patient population and under the real life conditions of a University hospital, perioperative treatment with BIC does not reduce the incidence of CSA-AKI as measured by postoperative changes in creatinine, and the need for dialysis. Moreover, it is associated with clearly unwarranted effects like a decrease in arterial blood pressure (during the bolus application of BIC), an increased need for fluids and vasopressors, and an increased need for treatment in the HDU. 
Table 6 Clinical course and complications

\begin{tabular}{|c|c|c|c|c|}
\hline & Total cohort & Control - no bicarbonate & Intervention - bicarbonate & Significance, $P$-value \\
\hline Number & 584 & $304(52.1 \%)$ & $280(47.9 \%)$ & \\
\hline Postoperative ventilation time, $h$ & $7(7,7)$ & $7(6,8)$ & $7(7,8)$ & 0.234 \\
\hline Reintubation & $46(7.9 \%)$ & $22(7.2 \%)$ & $24(8.6 \%)$ & 0.648 \\
\hline Tracheostomy & $18(3.1 \%)$ & $8(2.6 \%)$ & $11(3.6 \%)$ & 0.677 \\
\hline Duration in ICU, $h$ & $23(23,24)$ & $23(22,24)$ & $24(23,24)$ & 0.093 \\
\hline Duration in IMC, $h$ & $38(27,43)$ & $27(24,42)$ & $44(36,48)$ & 0.0008 \\
\hline Duration in HDU, $h$ & $85(73,89)$ & $83(71,88)$ & $88(72,96)$ & 0.026 \\
\hline Re-admission to ICU & 19 (3.3\%) & $9(3.0 \%)$ & $10(3.6 \%)$ & 0.849 \\
\hline Need for CPR & $10(1.7 \%)$ & $6(2.0 \%)$ & $4(1.4 \%)$ & 0.851 \\
\hline Reoperation & $80(13.7 \%)$ & $45(14.9 \%)$ & $35(12.5 \%)$ & 0.492 \\
\hline \multicolumn{5}{|l|}{ MAC Score } \\
\hline 1 & $54(9.2 \%)$ & $25(8.2 \%)$ & $29(10.4 \%)$ & 0.728 \\
\hline 2 & $25(4.3 \%)$ & $14(4.6 \%)$ & $11(3.9 \%)$ & \\
\hline 3 & $10(1.7 \%)$ & $5(1.6 \%)$ & $5(1.8 \%)$ & \\
\hline 4 & $1(0.2 \%)$ & $0(0.0 \%)$ & $1(0.4 \%)$ & \\
\hline \multicolumn{5}{|l|}{ Status upon discharge from hospital } \\
\hline Extubated & $551(94.3 \%)$ & $290(95.4 \%)$ & $261(93.2 \%)$ & 0.377 \\
\hline Intubated & $10(1.7 \%)$ & $2(0.7 \%)$ & $8(2.9 \%)$ & \\
\hline Tracheostomy & $13(2.2 \%)$ & $7(2.3 \%)$ & $6(2.1 \%)$ & \\
\hline Died in hospital & $8(1.4 \%)$ & $4(1.3 \%)$ & $4(1.4 \%)$ & \\
\hline \multicolumn{5}{|l|}{ Status after 30 days } \\
\hline Dead & $11(1.9 \%)$ & $5(1.6 \%)$ & $6(2.1 \%)$ & 0.573 \\
\hline Alive & $572(97.9 \%)$ & $298(98.0 \%)$ & $274(97.9 \%)$ & \\
\hline Unknown & $1(0.2 \%)$ & $1(0.3 \%)$ & $0(0.0 \%)$ & \\
\hline
\end{tabular}

Postoperative course, complications, and clinical outcomes are shown in patients undergoing elective on-pump cardiac surgery with or without a perioperative infusion of bicarbonate. CPR: cardiopulmonary resuscitation; MAC score: 1 point for each of the following complications - low cardiac output syndrome, stroke, new need for renal replacement therapy, reintubation; IMC: intermediate care unit; HDU: high dependency unit. Data are given as absolute numbers (percentage) for nominal variables, median ( $95 \%$ confidence interval) for non-normally distributed data, and mean (95\% confidence interval) for normally distributed variables.

The BIC dose chosen at our institution was comparable to the dose used by Haase and coworkers in their pilot study [7]. However, they did not observe any adverse effects or differences in the perioperative use of fluids. Unfortunately, the doses of vasopressors and inotropes used are not presented in this study.

With respect to the observational nature of our study, we cannot completely rule out the possibility that the adverse effects observed during the intervention period were related to other, unmeasured variables. However, the patient groups were ideally matched at baseline; not only with respect to conventional demographic factors and surgical procedures but also regarding the plasma levels of established cardiovascular risk markers. The patients were closely followed during the perioperative course, and a relevant number of patients were monitored invasively with a pulmonary arterial catheter to determine the cardiac index and $\mathrm{SvO} 2$. We are also not aware of any other clinical factor that was changed during this time: neither the introduction of another, new clinical treatment nor major changes in the treatment team. Thus it is rather likely that the observed adverse effects in the intervention period were indeed related to the use of bicarbonate infusion, that is, alkalization, despite only achieving minimal changes in maximal plasma $\mathrm{pH}$.

Data show that BIC is frequently used in cardiac surgical patients to treat acidosis, especially during cardiopulmonary bypass. Despite this, few data are available on the short- and long-term hemodynamic effects of BIC. Tripathi and coworkers observed a biphasic response after the infusion of $1 \mathrm{mmol} \mathrm{BIC} / \mathrm{Kg} \mathrm{BW}$ during steady state conditions during $\mathrm{CPB}$ with an immediate venous pooling (leading to a decrease in the $\mathrm{CPB}$ reservoir volume), followed by a moderate increase in MAP. In contrast, observations in patients with end-stage renal disease show that higher dialysate bicarbonate concentrations lead to a decrease in arterial blood pressure during dialysis [15], an effect that may be explained by an increase in endothelial nitric oxide production [16].

Since the differences in maximal $\mathrm{pH}$ between the study groups were rather small $(0.1 \mathrm{pH}$ difference) despite being statistically significant, one may assume that the lack of a nephroprotective effect of bicarbonate infusion may be related to the fact that no adequate alkalization was achieved. It is of note that Haase and coworkers observed a mean difference in $\mathrm{pH}$ of 0.7 
(7.38 to 7.45) [7] between the intervention and the control group with the same dose of bicarbonate. This discrepancy may be related to the fact that we did not record mean $\mathrm{pH}$ in the intra- and postoperative period, which would probably have better reflected the differences in this variable in comparison between the control and the intervention group.

As an important difference in comparison with the previous pilot study [7] we did not specifically treat only patients at risk for CSA-AKI, since the identification of such patients is not trivial with respect to the multifactorial nature of this complication. Median CPB duration, an accepted risk factor for CSA-AKI, was relatively long in the present study population, suggesting that our patients may be regarded as at risk per se. Nonetheless, the risk for AKI attributable to prolonged $\mathrm{CPB}$ is probably lower than the risk of selected groups of patients presenting with multiple risk factors for AKI. Consequently our findings do not rule out the possibility that a perioperative BIC infusion may be nephroprotective in selected, especially vulnerable cardiac surgery patients, as are currently being recruited for the BIC-NC study.

Comparably, we cannot rule out that the minor effect of the BIC infusion on plasma $\mathrm{pH}$ in our study in comparison with Haase's work may be related to the different types of crystalloid infusions used: $0.9 \%$ sodium chloride in the Australian setting [7] and a balanced electrolyte infusion containing $24 \mathrm{mmol} / \mathrm{L}$ actetate in our study. Thus, one may speculate that the use of balanced fluids may reduce the effectiveness of BIC as a measure to prevent CSA-AKI, in comparison with the clinical setting in Australia in which patients were treated with more acidic solutions [7]. Interestingly, acetate itself does also have vasodilating properties [17]. Consequently, the higher doses of crystalloid solutions used in the intervention group may also have aggravated the vasodilatation (that is, the higher need for vasopressors) observed in the intervention period.

\section{Limitations}

Despite all efforts to follow the patients meticulously and to rule out other factors that may have influenced our results, the design of this study as an observational cohort study has a substantial risk of bias. The present ongoing BIC-NC study (Clinical trials identifier NCT00672334) will help to elucidate further, the risks and benefits of using BIC to prevent AKI in patients undergoing cardiac surgery.

With respect to the observational nature of this study we did not perform an a priori power analysis to determine if the sample size is adequate. However, we enrolled more patients than planned for the BIC-NC study and additionally tested the endpoint, namely, $25 \%$ increase in plasma creatinine, suggesting that our dataset is adequately powered.

\section{Conclusion}

In conclusion, the present prospective observational trial failed to reproduce the beneficial effects of a 24-h perioperative infusion of $4 \mathrm{mmol} / \mathrm{Kg} \mathrm{BIC}$ on the incidence of AKI in patients undergoing cardiac surgery, that were recently shown in a small pilot trial. Routine treatment in a heterogeneous cohort with BIC was associated with an increased need for fluids and vasopressors. If this is a direct effect of BIC infusion, or the indirect effect of an associated treatment with balanced crystalloid solutions containing the vasodilating buffer acetate, merits further investigation.

\section{Key messages}

- The routine perioperative infusion of $4 \mathrm{mmol} / \mathrm{Kg}$ BW sodium failed to reduce the incidence of acute kidney injury and the need for renal replacement therapy in a heterogeneous cohort of cardiac surgical patients.

- Treatment with BIC was associated with hypotension, a higher need for vasopressors, and a prolonged stay in the HDU but had no other significant adverse effects on non-renal morbidity and 30-day mortality.

\section{Abbreviations}

AKI: acute kidney injury; BIC: sodium bicarbonate; CABG: coronary artery bypass grafting; Cl: cardiac index; CON: control (non-intervention) group; CPB: cardiopulmonary bypass; CSA-AKI: cardiac surgery-assocaiated acute kidney injury; CVP: central venous pressure; eGFR: estimated glomerular filtration rate; HDU: high dependency unit; HR: heart rate; hsTNT: highsensitivity troponin $T_{\text {; }} I C U$ : intensive care unit; IMC: intermediate care unit; MAP: mean arterial pressure; NTproBNP: N-terminal pro B-type natriuretic peptide; PAP: pulmonary arterial pressure; RRT: renal replacement therapy; ScO2: cerebral oxygen saturation; ScvO2: central venous oxygen saturation; SvO2: mixed venous oxygen saturation.

\section{Acknowledgements}

This work was supported by institutional funding. Plasma levels of NTproBNP and hsTNT were kindly determined by Roche Diagnostics, Mannheim, Germany. We deeply thank the nursing staff of the cardiac anesthesia unit, the intensive care unit 15i, and the cardiac surgical ward 19 of the University of Lübeck for their help in performing this study.

\section{Author details}

'Department of Anesthesiology, University of Lübeck, Ratzeburger Allee 160, Lübeck, D-23538, Germany. ${ }^{2}$ Department of Cardiac and Thoracic Vascular Surgery, University of Lübeck, Ratzeburger Allee 160, Lübeck, D-23538, Germany.

\section{Authors' contributions}

$\mathrm{MH}, \mathrm{HH}, \mathrm{JH}$ and $\mathrm{TH}$ designed the study, supervised the statistical analyses and drafted the manuscript. MS, YN, JG, MK, and HP collected the data and performed the statistical analyses. All authors read and approved the final manuscript.

\section{Competing interests}

The authors declare that they have no competing interests. 


\section{References}

1. Heringlake M, Knappe M, Vargas Hein O, Lufft H, Kindgen-Milles D, Böttiger BW, Weigand MR, Klaus S, Schirmer U: Renal dysfunction according to the ADQI-RIFLE system and clinical practice patterns after cardiac surgery in Germany. Minerva Anestesiol 2006, 72:645-654.

2. Stafford-Smith M, Shaw A, Swaminathan M: Cardiac surgery and acute kidney injury: emerging concepts. Curr Opin Crit Care 2009, 15:498-502.

3. Hobson CE, Yavas S, Segal MS, Schold JD, Tribble CG, Layon AJ, Bihorac A: Acute kidney injury is associated with increased long-term mortality after cardiothoracic surgery. Circulation 2009, 119:2444-2453.

4. Ronco C, Kellum JA, Bellomo R: Cardiac surgery-associated acute kidney injury. Int J Artif Organs 2008, 31:156-157.

5. Haase M, Haase-Fielitz A, Bagshaw SM, Ronco C, Bellomo R: Cardiopulmonary bypass-associated acute kidney injury: a pigment nephropathy? Contrib Nephrol 2007, 156:340-353.

6. Bosch X, Poch E: Rhabdomyolysis and Acute Kidney Injury. N Engl J Med 2009, 361:62-72

7. Haase M, Haase-Fielitz A, Bellomo R, Devarajan P, Story D, Matalanis G, Reade MC, Bagshaw SM, Seevanayagam N, Seevanayagam S, Doolan L, Buxton B, Dragun D: Sodium bicarbonate to prevent increases in serum creatinine after cardiac surgery: a pilot double-blind, randomized controlled trial. Crit Care Med 2009, 37:39-47.

8. Carl M, Alms A, Braun J, Dongas A, Erb J, Goetz A, Goepfert M, Gogarten W, Grosse J, Heller AR, Heringlake M, Kastrup M, Kroener A, Loer SA, Marggraf G, Markewitz A, Reuter D, Schmitt DV, Schirmer U, Wiesenack C, Zwissler B, Spies C: S3 guidelines for intensive care in cardiac surgery patients: hemodynamic monitoring and cardiocirculary system. Ger Med Sci 2010, 8:Doc12.

9. Mehta RL, Kellum JA, Shah SV, Molitoris BA, Ronco C, Warnock DG, Levin A, Acute Kidney Injury Network: Acute Kidney Injury Network: report of an initiative to improve outcomes in acute kidney injury. Crit Care 2007, 11 R31.

10. Chertow GM, Levy EM, Hammermeister KE, Grover F, Daley J: Independent association between acute renal failure and mortality following cardiac surgery. Am J Med 1998, 104:343-348.

11. Lassnigg A, Schmidlin D, Mouhieddine M, Bachmann LM, Druml W, Bauer P, Hiesmayr M: Impact of minimal increases in serum creatinine on outcome in patients after cardiothoracic surgery: Do we have to revise current definitions of acute renal failure? Crit Care Med 2008, 36:1129-1137.

12. Vanholder R, Sever M, Erek E: Rhabdomyolysis. J Am Soc Nephrol 2000, 11:1553-1561.

13. Schetz M, Bove T, Morelli A, Mankad S, Ronco C, Kellum JA: Prevention of cardiac surgery associated acute kidney injury. Int J Artif Organs 2008, 31:179-189.

14. Heringlake M, Kindgen-Milles D, Hackmann F, Haake N, Kielstein J, Lance M, Lufft H, Just S, Trabold B, Scherlitz A, Schirmer U, Schmitt D, Vargas-Hein O, Markewitz A: Pathophysiology, prophylaxis, and treatment of cardiac surgery-associated kidney injury. Position of the working group renal failure of the collaborative group Cardiothoracic Intensive Care of the DGAI and DGTHG on the consensus statement of the Acute Dialysis Quality Initiative. Z Herz-Thorax-Gefäßchir 2009, 23:349-373, [article in German language].

15. Gabutti L, Ferrari N, Giudici G, Mombelli G, Marone C: Unexpected haemodynamic instability associated with standard bicarbonate haemodialysis. Nephrol Dial Transplant 2003, 18:2369-2376.

16. Schulz E, Munzel T: Intracellular pH: a fundamental modulator of vascular function. Circulation 2011, 124:1806-1807.

17. Daugirdas JT, Nawab ZM, Ing TS, Leehey DJ, Yang MC, Pang PK, Klok MA: Studies of the vasorelaxant effect of acetate. Trans Am Soc Artif Intern Organs 1984, 30:65-68

doi:10.1186/cc11476

Cite this article as: Heringlake et al: A perioperative infusion of sodium bicarbonate does not improve renal function in cardiac surgery patients: a prospective observational cohort study. Critical Care 2012 16: R156.

\section{Submit your next manuscript to BioMed Central and take full advantage of:}

- Convenient online submission

- Thorough peer review

- No space constraints or color figure charges

- Immediate publication on acceptance

- Inclusion in PubMed, CAS, Scopus and Google Scholar

- Research which is freely available for redistribution 